\title{
Modulation of the intestinal microbiota of dogs by kefir as a functional dairy product
}

\author{
Dong-Hyeon Kim, ${ }^{1}$ Dana Jeong, ${ }^{1}$ II-Byeong Kang, ${ }^{1}$ Hyun-Woo Lim, ${ }^{1}$ YoonJu Cho, ${ }^{2}$ and Kun-Ho Seo ${ }^{1 *}$ \\ ${ }^{1}$ Center for One Health, College of Veterinary Medicine, Konkuk University, Seoul, 05029, South Korea \\ ${ }^{2}$ Department of Pet Science, Seojeong College, Yangju, 11429, South Korea
}

\section{ABSTRACT}

Kefir is a traditional dairy product with multiple probiotic characteristics derived from its associated microorganisms, including more than 50 species of lactic acid bacteria and yeast. For centuries, many people have produced kefir for human consumption; its consumption and potential role as a probiotic supplement in companion animals have never been tested. The present study explored the potential application of kefir as a probiotic supplement for dogs. Kefir was orally administered to healthy adult dogs $(n=6)$ for 2 wk. On d 0 and 14 (before and after kefir consumption, respectively), gut microbiota was analyzed comprehensively using quantitative $\mathrm{PCR}$ and $16 \mathrm{~S}$ rDNA amplicon-based community analysis using fresh fecal samples. The $16 \mathrm{~S}$ rDNA amplicon-based community analysis showed that the relative abundance of the phylum Fusobacteria was significantly decreased after kefir consumption. Furthermore, the relative abundance of the families Prevotellaceae, Selenomonadaceae, and Sutterellaceae increased significantly, whereas that of the families Clostridiaceae, Fusobacteriaceae, and Ruminococcaceae decreased significantly. The quantitative PCR assay showed that kefir consumption significantly increased the population of lactic acid bacteria and the lactic acid bacteria:Enterobacteriaceae ratio and significantly decreased the Firmicutes:Bacteroidetes ratio. In summary, 2-wk kefir administration successfully modified the gut microbiota without causing any clinically evident adverse effects. Therefore, kefir could be further developed as a novel probiotic food supplement for dogs to improve the quality of life of dogs.

Key words: kefir, gut microbiota, probiotic, community analysis, feed supplement

Received September 3, 2018.

Accepted December 30, 2018.

*Corresponding author: bracstu3@konkuk.ac.kr

\section{INTRODUCTION}

The intestinal microbiota encompasses a large variety of microorganisms in the intestinal tract, with at least $10^{14}$ microbial cells in mammalians, originating from up to 1,000 different species (Suchodolski, 2011). To date, gut microbiota and its association with health or disease have been assessed in various animals, including dogs (Suchodolski, 2011; Hooda et al., 2012; Strompfová et al., 2014). Owing to increasing interest in the health and welfare of companion animals, especially dogs, the composition and role of canine intestinal microbiota have been actively investigated, and studies on the possibilities of its therapeutic modification with various probiotics, prebiotics, and synbiotics have increased in the past decades (Schmitz and Suchodolski, 2016). For instance, 4 individual bacterial strains-Enterococcus faecium NCIMB10415, Ec. faecium NCIMB10415, Lactobacillus acidophilus DSM13241, and Bifidobacterium animalis ssp. animalis NCIMB41617-have been assessed by the European Food Safety Authority for their safety and efficacy as probiotics or food supplements for dogs (Schmitz and Suchodolski, 2016). Other species, including Lactobacillus fermentum, Butyrivibrio fibrisolvens, and Lb. acidophilus, have been actively investigated as potential probiotic agents (Asanuma et al., 2001; Beasley et al., 2006; Tang and Saris, 2013). However, these candidates were tested individually, whereas foods often contain multiple strains, and their efficacy in trials suggests that they should be manufactured in the pharmaceutical industry to reach consumers (Schmitz and Suchodolski, 2016).

Kefir is a traditional fermented milk containing more than 50 species of microorganisms, including lactic acid bacteria, yeast, and acetic acid bacteria (Guzel-Seydim et al., 2011). According to the Codex Standard for Fermented Milks (Codex Alimentarius, 2003), kefir is defined as fermented milk prepared from a unique starter culture known as kefir grains comprising lactic acid bacteria, acetic acid bacteria, and yeast. We previously reported that kefir administration for 3 wk successfully modulated gut microbiota in mice fed a normal diet 
(Kim et al., 2015b) and that regular kefir administration prevented obesity and nonalcoholic fatty liver disease induced by a high-fat diet by improving gut micro- and mycobiota (Kim et al., 2017b). In addition, individual kefir microorganisms, such as Lactobacillus kefiranofaciens DN1 and Lactobacillus kefiri DH5, and bioactive compounds such as exopolysaccharides could modulate the gut microbiota and exerted beneficial health effects (Jeong et al., 2017a,b; Kim et al., 2017a, 2018).

The application of human probiotics to dogs showed several benefits in some studies (Aktas et al., 2007; Rossi et al., 2014). However, no studies have investigated the beneficial effects of kefir on the gastrointestinal tract of dogs, especially on the gut microbiota. Considering interspecies differences in the anatomical, metabolic, and physiological aspects among the aforementioned animals, this study aimed to investigate the modulatory effect of kefir on the gut microbiota of healthy dogs and provide experimental information regarding the possibility of its use as a novel probiotic food supplement for dogs.

\section{MATERIALS AND METHODS}

\section{Kefir Preparation}

In total, $50 \mathrm{~g}$ of viable kefir grain (Sensorgen Co., Seoul, South Korea) was used to inoculate $500 \mathrm{~mL}$ of sterilized milk (Seoul Milk Inc., Seoul, South Korea) and was cultured at $25^{\circ} \mathrm{C}$ for $24 \mathrm{~h}$. After fermentation, the grain and milk were separated using a sterilized plastic filter (2-mm pore size). Kefir milk was prepared daily during the experimental period. Lactic acid bacteria and yeast in kefir milk were enumerated in accordance with our pervious study (Kim et al., 2015a). Kefir milk contained $9.32 \pm 0.23 \mathrm{log} \mathrm{cfu} / \mathrm{mL}$ of lactic acid bacteria and $7.12 \pm 0.36 \log \mathrm{cfu} / \mathrm{mL}$ of yeast.

\section{Animals and Experimental Designs}

Healthy adult dogs $[\mathrm{n}=6 ; 4$ male, 2 female; mean age $=5.17 \pm 2.32$ yr (range: $3-8$ ); mean $\mathrm{BW}=28.65$ $\pm 2.94 \mathrm{~kg}$ (range: $24.2-32.0$ )] of the following breeds were included in the present study: mongrel, Poodle, Shepherd $(\mathrm{n}=2)$, Malinois, and Labrador. The experimental design was approved by the Seojeong College Institutional Animal Care and Use Committee (SJ2017-04). Dogs were individually housed in floor pens $\left(2.5 \mathrm{~m}^{2}\right)$ and provided outdoor exercise once a day. The animals were kept at ambient temperatures between 20 and $24^{\circ} \mathrm{C}$ in a room with a humidity of 30 to $70 \%$ and natural light. Dogs were fed extruded dry dog food twice daily according to the recommended daily feeding portion (Natural Balance Ultra Premium, Dick Van Patten's Natural Balance Pet Foods, Burbank, CA). Water was provided ad libitum. Environmental enrichment was provided in the form of sanitized toys, food treats, and social interaction with humans. The adaptation period to this food was 4 wk before the experiment. The 14-d experiment comprised a kefir preconsumption phase (d 0) and postconsumption phase (d 14). During the experimental period, dogs were provided with $200 \mathrm{~mL}$ of kefir once a day and consumed it ad libitum. Food intake, water consumption, and kefir consumption were recorded daily for individual dogs. On d 0 and 14, changes in BW, BCS, fecal quality, hematological parameters, and gut microbiota of the dogs were monitored. Body condition score was determined as follows: 1 = very thin (bones such as ribs could be easily observed and palpated with no fat cover), $2=$ underweight (bones could be palpated with a little amount of fat cover), $3=$ ideal (ribs can be palpated through slight fat cover), $4=$ overweight (difficult to palpate ribs due to moderate fat cover), and $5=$ obese (difficult to palpate ribs due to thick fat cover).

\section{Sampling Procedures}

Fresh fecal samples were collected on d 0 (before kefir consumption) and d 14 (after kefir consumption). Bristol's fecal score was determined immediately and fecal samples were stored at $4^{\circ} \mathrm{C}$ less than $2 \mathrm{~h}$ before DNA extraction (Lewis and Heaton, 1997). Blood samples were collected via venipuncture from the vena cephalica antebrachii on d 0 and 14 in heparin-coated tubes for hematological analysis.

\section{Fecal Quality Analysis}

Fecal samples were scored in accordance with Bristol's scoring guidelines (Lewis and Heaton, 1997): $1=$ separate hard lumps; $2=$ lumpy and sausage-like; $3=$ a sausage shape with cracks on the surface; $4=$ like a smooth, soft sausage or snake; $5=$ soft blobs with clear-cut edges; $6=$ mushy consistency with ragged edges; and $7=$ liquid consistency with no solid pieces. One gram of each fecal sample was used to determine fecal water content after drying the fecal samples for $24 \mathrm{~h}$ in an $80^{\circ} \mathrm{C}$ dry oven using the following equation: [ffecal weight before drying - fecal weight after drying)/feces weight before drying] $\times 100$. Finally, fecal occult blood was assessed using a Hema-Screen Lab Pack (Stanbio Laboratory, Boerne, TX) in accordance with the manufacturer's instructions. 


\section{Hematological Analysis}

Hematological parameters were analyzed using an Advia 2120 hematology analyzer (Siemens Healthcare Diagnostics, Deerfield, IL) in accordance with the manufacturer's instructions.

\section{DNA Extraction from Fecal Samples}

Genomic DNA was extracted using a NucliSENS easyMAG instrument (bioMérieux, Marcy l'Etoile, France) in accordance with the manufacturer's instructions. Briefly, each fecal sample was inoculated in $1 \mathrm{~mL}$ of lysis buffer containing guanidine thiocyanate, Triton $\mathrm{X}-100$, and EDTA and was homogenated vigorously by vortexing and chopping. The mixture was incubated overnight at $25^{\circ} \mathrm{C}$. The lysed sample was then transferred to the well of a plastic vessel with $50 \mu \mathrm{L}$ of magnetic silica and subjected to automatic magnetic bead separation. The DNA was then resuspended in $50 \mu \mathrm{L}$ of elution buffer.

\section{Gut Microbiota Analysis}

Each sequenced sample was prepared in accordance with the Illumina (San Diego, CA) 16S Metagenomic Sequencing Library protocols. The DNA was quantified and its quality was assessed using PicoGreen (Molecular Probes, Eugene, OR) and Nanodrop (Thermo Fisher Scientific, Waltham, MA). The $16 \mathrm{~S}$ rDNA was amplified using $16 \mathrm{~S}$ V3-V4 primers. The primer sequences are as follows: $16 \mathrm{~S}$ amplicon PCR forward primer: 5'-TCGTCGGCAGCGTCAGATGTGTATAAGAGACAGCCTACGGGNGGCWGCAG-3'; $16 \mathrm{~S}$ amplicon PCR reverse primer: 5'-GTCTCGTGGGCTCGGAGATGTGTATAAGAGACAGGACTACHVGGGTATCTAATCC-3'. The PCR reaction was performed with Herculase II Fusion DNA Polymerase (Agilent Technologies, Santa Clara, CA). The reaction for amplicon PCR was performed at $95^{\circ} \mathrm{C}$ for $3 \mathrm{~min}$, followed by 25 cycles each at $95^{\circ} \mathrm{C}$ for $30 \mathrm{~s}, 55^{\circ} \mathrm{C}$ for $30 \mathrm{~s}$, and $72^{\circ} \mathrm{C}$ for $30 \mathrm{~s}$, and then at $72^{\circ} \mathrm{C}$ for $5 \mathrm{~min}$. For index PCR, the cycle was at $95^{\circ} \mathrm{C}$ for $3 \mathrm{~min}$, followed by 10 cycles each at $95^{\circ} \mathrm{C}$ for $30 \mathrm{~s}, 55^{\circ} \mathrm{C}$ for $30 \mathrm{~s}$, and $72^{\circ} \mathrm{C}$ for $30 \mathrm{~s}$, and then at $72^{\circ} \mathrm{C}$ for $5 \mathrm{~min}$. Thereafter, a subsequent limited-cycle amplification step (301 cycles $\times 2$ ) was performed to add multiplexing indices and Illumina sequencing adapters. The final products were normalized and pooled using PicoGreen, and the size of libraries was verified using the LabChip GX HT DNA High Sensitivity Kit (PerkinElmer, Waltham, MA). Thereafter, the DNA fragment (120-160 bp) was sequenced using the MiSeq platform (Illumina) from both ends.
After sequencing analysis of MiSeq raw data, a FASTQ file was generated using MCS (MiSeq Control Software version 2.2) and bcl2fastq (version 1.8.4), and a PhiX sequence was obtained through BurrowsWheeler Aligner (BWA). High-quality sequences with a Phred quality score of 25 or better were selected. By merging paired-end MiSeq forward and reverse reads ( $\sim 300$ bp, respectively), the single long reads ( $\sim 40$ to $465 \mathrm{bp}$ ) were obtained using FLASH (1.2.11; Magoč and Salzberg, 2011). The obtained sequence was processed using the CD-HIT-EST-based operational taxonomic unit (OTU) analysis program CD-HIT-OTU (Li et al., 2012) to eliminate low-quality, ambiguous, and chimeric sequences, which were considered sequencing errors, clustering to form species-level OTU at clustering cutoff of $97 \%$.

The representative sequence of each OTU was determined using BLASTN (version 2.4.0; Zhang et al., 2000) in Reference DB (NCBI 16S Microbial), and taxonomic assignment was performed using information regarding the most similar subject. If the query coverage of the best hit that matched the database was $<85 \%$ and the identity of the matched domain was $<85 \%$, the taxon was considered undefined. The sequences were identified to phylum level at $\geq 85 \%$, genus level at $\geq 94 \%$, and species level at $\geq 97 \%$ similarity.

Various comparisons within the fecal community were performed using QIIME (version 1.8; Caporaso et al., 2010) and considered OTU information. Shannon and inversed Simpson indices were obtained to confirm species diversity and evenness of microbial communities in fecal samples. Alpha diversity was determined via a rarefaction curve and Chao1 value. The principal coordinate analysis based on weighted UniFrac distance was conducted to visualize the community diversity. The hierarchical relationship between samples was visualized via construction of an unweighted pair group method with arithmetic mean phylogenetic tree.

The quantity of total bacteria, yeast, Firmicutes, Bacteroidetes, lactic acid bacteria, Bifidobacterium, Enterobacteriaceae, and Clostridium cluster I was determined from fecal-derived DNA samples using quantitative PCR (qPCR) analysis per our previous reports (Supplemental Table S1, https://doi.org/10.3168/jds .2018-15639; Kim et al., 2015b, 2017a,b; Jeong et al., 2017b).

\section{Statistical Analysis}

All data are presented as the mean \pm standard deviation. Statistical analyses were conducted using SPSS version 18.0 (SPSS Inc., Chicago, IL). All data obtained from samples including relative abundances of fecal microbiota were tested for normality using the 
Shapiro-Wilk normality test. A paired $t$-test was used to evaluate statistical significance between d 0 and 14 . A $P$-value less than 0.05 was considered statistically significant.

\section{RESULTS}

\section{Change in BW and BCS}

No significant changes were observed in BW and BCS before and after kefir consumption (Table 1). Weight loss greater than $15 \%$ of initial BW was not observed in any dogs during the experimental period, suggesting that kefir is not detrimental to canine health.

\section{Food and Water Intake}

During the experimental period, dogs consumed more than $94 \%$ of the provided kefir between 1 and 3 min actively and spontaneously, suggesting that kefir is a potentially suitable natural food supplement or can be used as a treat to attract attention (Supplemental Table S2, https://doi.org/10.3168/jds.2018-15639). In addition, kefir consumption did not affect the dietary habits of the experimental dogs (Supplemental Table S2). All dogs consumed the entire amount of provided food except for $\operatorname{dog} 2$, who consumed $89.4 \%$ of the provided food. Dogs drank water normally throughout the experimental period, which was demonstrated by dehydration pinch test.

\section{Fecal Quality}

Two weeks of kefir consumption significantly increased fecal water content, and Bristol fecal scores remained unchanged (Table 1). In addition, occult blood was absent in the feces of all dogs, suggesting that kefir is not harmful to the gastrointestinal tract of dogs (Table 1).

\section{Hematological Parameters}

Hematological parameters of the experimental dogs were within the physiological reference ranges before and after kefir consumption, indicating that kefir consumption is not associated with the risk of subsequent anemia, systemic inflammation, or coagulative disorders (Supplemental Table S3, https://doi.org/10.3168/ jds.2018-15639).

\section{Modulation of Gut Microbiota}

Figure 1 illustrates the results of next-generation sequencing (NGS) analysis of canine gut microbiota
Table 1. Physical examination and fecal parameters of dogs on $\mathrm{d} 0$ and $14^{1}$

\begin{tabular}{lcc}
\hline Parameter & $\mathrm{d} 0$ & $\mathrm{~d} 14$ \\
\hline BW $(\mathrm{kg})$ & $28.65 \pm 2.94^{\mathrm{a}}$ & $28.28 \pm 2.63^{\mathrm{a}}$ \\
BCS $^{2}$ & $3^{\mathrm{a}}$ & $3^{\mathrm{a}}$ \\
Fecal water contents $(\%)$ & $62.51 \pm 4.14^{\mathrm{a}}$ & $70.70 \pm 1.73^{\mathrm{b}}$ \\
Bristol score & $3.5 \pm 0.84^{\mathrm{a}}$ & $3.67 \pm 0.52^{\mathrm{a}}$ \\
Occult blood in feces & $0 / 6$ & $0 / 6$ \\
\hline${ }^{\mathrm{a}, \mathrm{b}}$ Different letters in a row indicate a significant difference $(P<0.05$ \\
by paired and 2-way Student's $t$-test). \\
${ }^{1}$ Data are expressed as mean \pm SD. \\
${ }^{2} 1$ = very thin; $2=$ underweight; $3=$ ideal; 4 = overweight; $5=$ obese.
\end{tabular}

assessed via $16 \mathrm{~S}$ rDNA amplicon-based community analysis before and after 2 wk of kefir consumption. In principal coordinate analysis based on weighted UniFrac distance, the fecal microbiota after kefir consumption was not fully separate from that before kefir consumption, although fecal microbiota of dogs after kefir consumption was roughly clustered (Figure 1A). Similarly, hierarchical clustering analysis via construction of an unweighted pair group method with arithmetic mean tree indicated an insignificant difference in fecal microbiota before and after kefir consumption (Figure 1B).

To evaluate the richness of gut microbiota, we compared the Chao1, Shannon, and Simpson diversity indices of the canine fecal samples obtained before and after kefir consumption (Figure 1C). There were no significant differences in the Chao1, Shannon, and Simpson indices, indicating that richness and evenness were similar before and after kefir consumption. Good's coverage values were $>0.999$ for fecal samples obtained before and after kefir consumption, indicating negligible undetected diversity (Figure 1C).

Gut microbiota composition before and after kefir consumption was compared at the phylum and family levels, respectively (Figure 1D and 1E; Supplemental Tables S4 and S5, https://doi.org/10.3168/jds.2018 -15639). At the phylum level, the most significant differences were observed in Fusobacteria $(P=0.014$; Figure 1D, Supplemental Table S4). At the family level, the relative abundances of Prevotellacea, Selenomonadaceae, and Sutterellaceae increased significantly $(P=$ $0.027,0.044$, and 0.028 , respectively), whereas those of Clostridiaceae, Fusobacteriaceae, and Ruminococcaceae decreased significantly $(P=0.039,0.014$, and 0.034 , respectively) following kefir consumption (Figure $1 \mathrm{E}$ and Supplemental Table S5). Species-level alterations in the gut microbiota are represented as a heatmap (Figure $1 \mathrm{~F})$. In total, 92.55 to $94.12 \%$ of obtained sequences were accurately identified to the bacterial species level. The relative abundance of Bacteroides uniformis, Fusobacterium perfoetens, Fecalibacterium prausnitzii, 
Fusobacterium mortiferum, Pseudoflavonifractor capillosus, and Fusicatenibacter saccharivorans decreased significantly, whereas that of Catenibacterium mitsuokai increased significantly after kefir consumption $(P<$ 0.05 , Figure $1 \mathrm{~F})$.
In addition, group-specific qPCR analysis of gut microbiota harvested from fecal samples after 2 wk of kefir consumption was conducted for certain microbes of interest to complement the results of the NGSbased analysis (Figure 2). Kefir consumption signifi-
(A) 3D PCoA plot

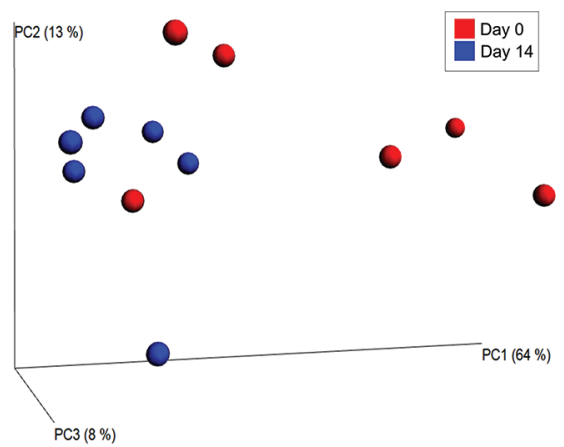

(B) UPGMA tree

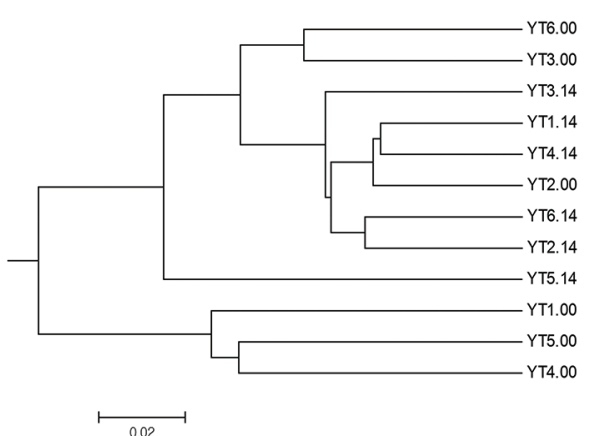

(C) Diversity Index

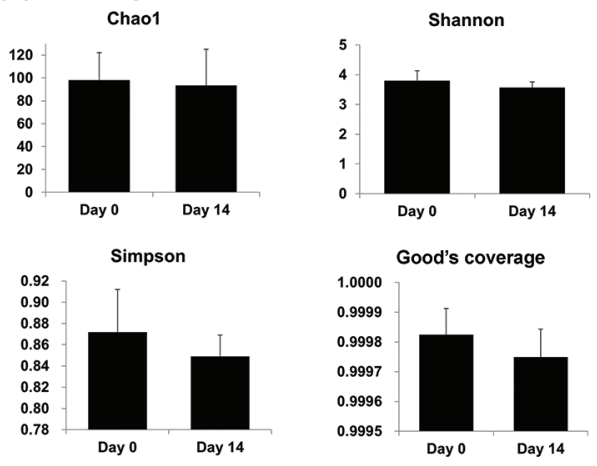

(D) Phylum level

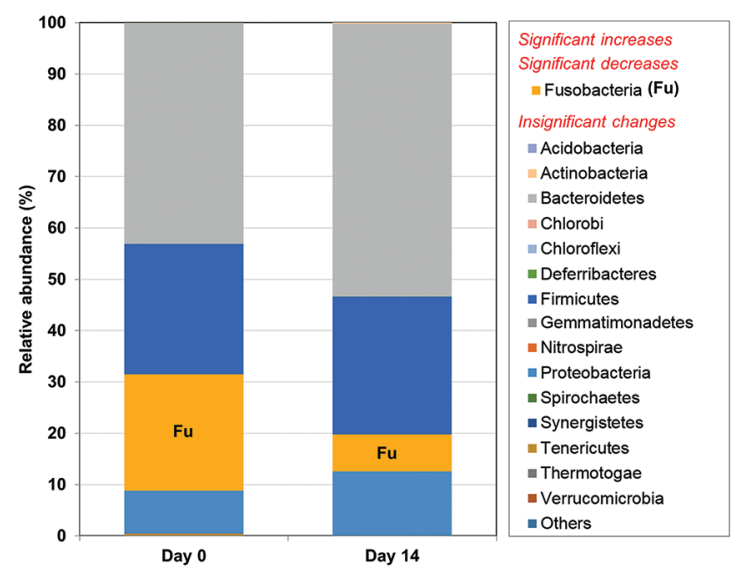

(F) Species level - Heatmap analysis

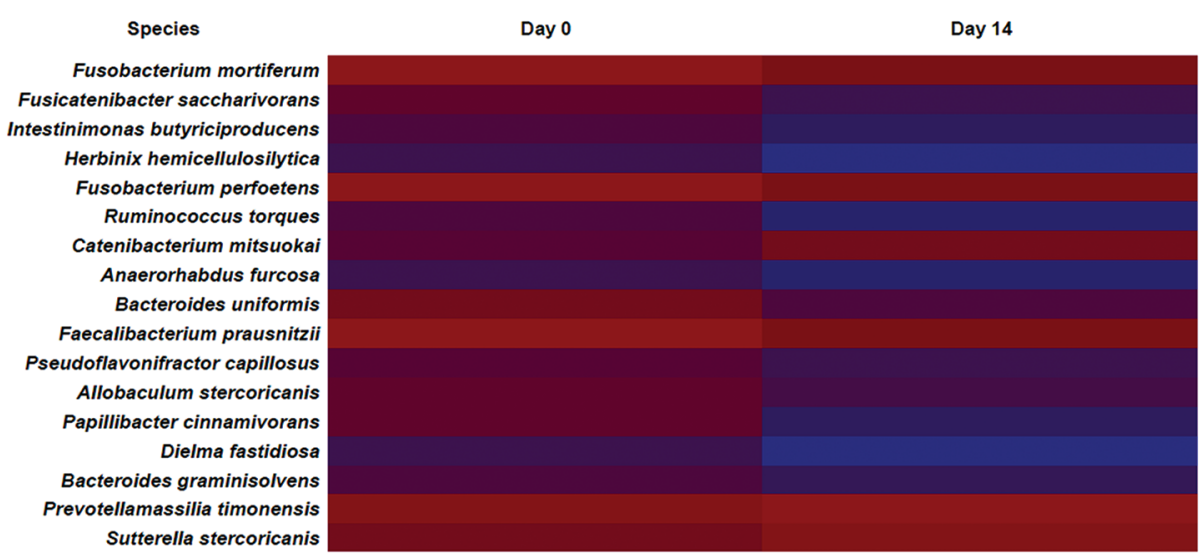

Day 0
(E) Family level
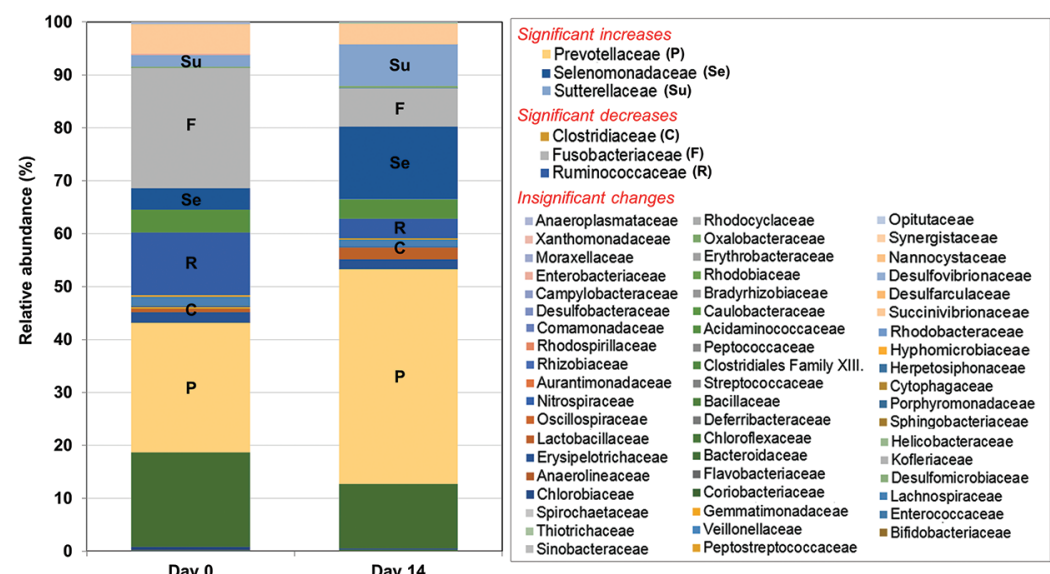

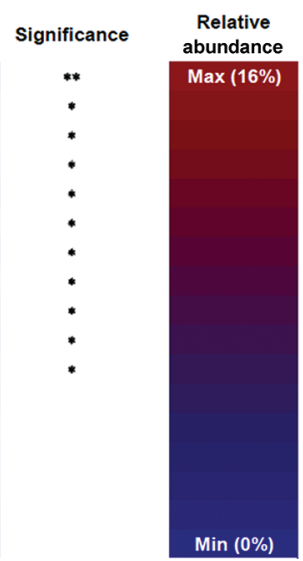

Figure 1. Effect of 2-wk kefir consumption on the intestinal microbiota of healthy adult dogs analyzed via the $16 \mathrm{~S}$ rDNA amplicon-based community analysis. The analysis was performed using fecal samples collected at d 0 and $14(\mathrm{n}=6 / \mathrm{d})$. (A) Principal coordinate analysis (PCoA) based on weighted UniFrac distance represented in the 3-dimensional plot; (B) unweighted pair-group method using arithmetic averages (UPGMA) clustering; (C) diversity indices; (D, E) relative abundances of gut microbiota at the (D) phylum and (E) family levels; and (F) heatmap analysis of gut microbiota at the species level. The color of the reference bar represents a relative abundance. $P$-value $<0.05(*)$ and $<0.01(* *)$ was considered significant. 
cantly increased the population of lactic acid bacteria $(P=0.012)$, significantly decreased the Firmicutes: Bacteroidetes ratio $(P=0.026)$, and significantly increased the lactic acid bacteria:Enterobacteriaceae ratio $(P=0.044)$. These were consistent with the results obtained by NGS analysis (the relative abundance of Firmicutes changed from 25.40 to $26.92 \%$, that of Bacteroidetes changed from 42.99 to $53.01 \%$, that of Lactobacillaceae changed from 0.62 to $2.18 \%$, and that of Enterobacteriaceae changed from 0.25 to $0.03 \%$ ). The number of total bacteria was not changed by 2 wk of kefir consumption.

\section{DISCUSSION}

The present study reports that 2 wk of kefir administration can successfully modulate the gut microbiota of healthy adult dogs without resulting in any clinically evident adverse effects. Kefir has been assessed extensively as a potential probiotic food supplement in human and animal models of disease (Bourrie et al., 2016; Kim et al., 2018); however, it has not been investigated in dogs. The probiotic attributes of beneficial microorganisms, such as survivability and functional- ity, cannot be directly extrapolated from one species to another. Therefore, we examined, for the first time, the applicability of kefir as a novel probiotic food supplement for dogs.

For decades, numerous studies have investigated the application of probiotics in dogs to improve their health and to control diseases (Weese and Anderson, 2002; Manninen et al., 2006; Wynn, 2009; Silva et al., 2013). For instance, numerous studies isolated lactic acid bacteria from canine feces to develop novel probiotic agents for dogs, such as Lb. fermentum AD1, Enterococcus faecalis EE4, Ec. faecium EF01, Lb. fermentum LAB8, Lactobacillus salivarius LAB9, Lactobacillus rhamnosus LAB11, and Lactobacillus mucosae LAB12 (Strompfová et al., 2004, 2006; Manninen et al., 2006). These strains have been shown to colonize the canine gastrointestinal tract (Strompfová et al., 2004), decrease blood glucose levels (Strompfová et al., 2006), increase serum protein levels (Strompfová et al., 2006), and compete with enteropathogenic microorganisms (Rinkinen et al., 2003). Furthermore, gut microbiota is closely associated with clinical canine gastrointestinal disorders such as inflammatory bowel disease and small-intestinal bacterial overgrowth and systemic diseases such as
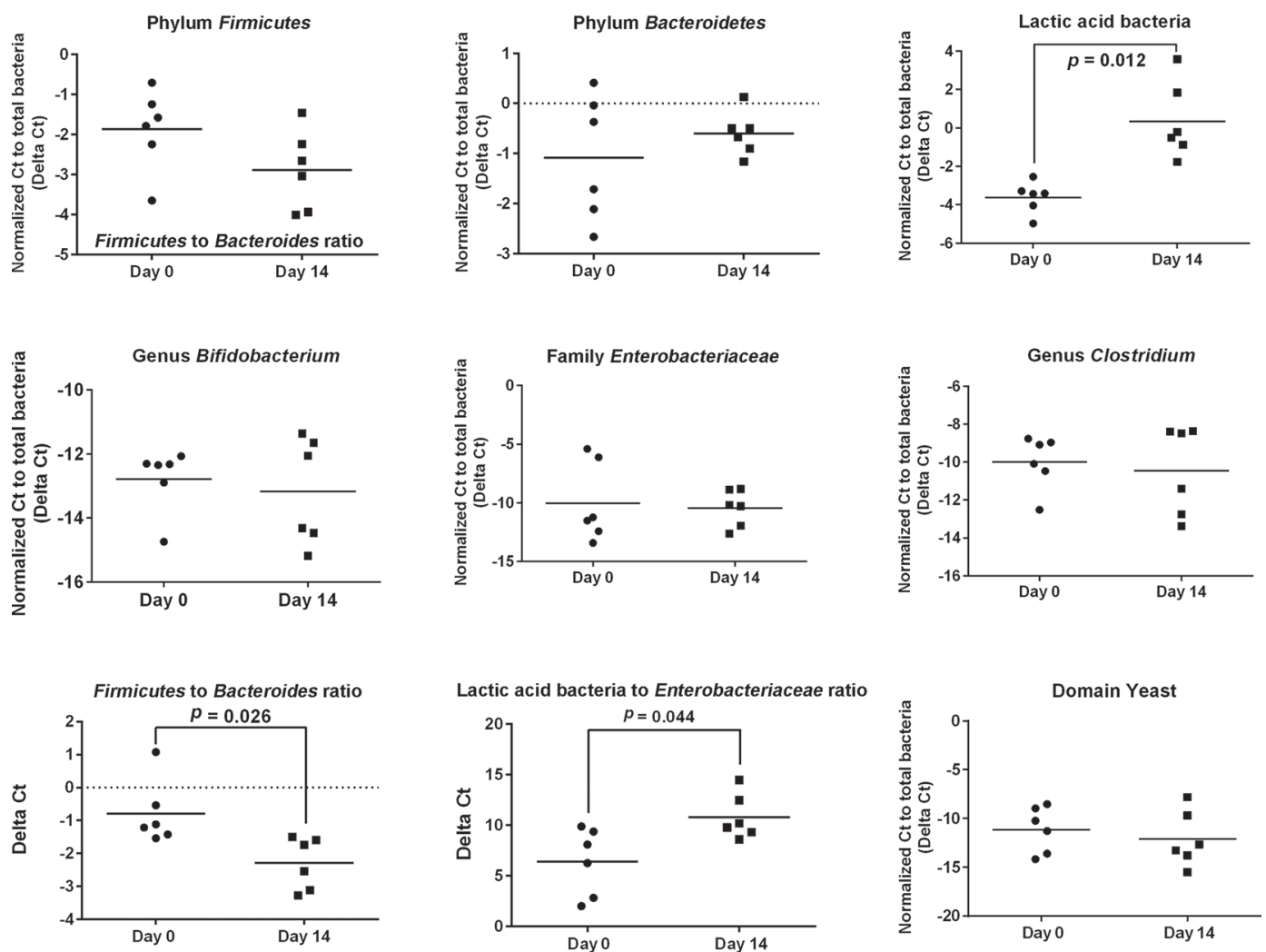

Figure 2. Effect of 2-wk kefir consumption on the intestinal microbiota of healthy adult dogs analyzed via quantitative real-time PCR Highlighted taxa comprise total bacteria (used as a normalization factor), yeast, Firmicutes, Bacteroidetes, lactic acid bacteria, Bifidobacterium, Enterobacteriaceae, and Clostridium. Ct = cycle threshold. 
obesity and immune-related disorders (Manninen et al., 2006; Suchodolski and Simpson, 2013; Schmitz and Suchodolski, 2016). To prevent these diseases, pet foods have been formulated with various probiotic bacteria, although the actual viable count of these microorganisms does not generally amount to the labeled descriptions, which prevents the food from having its intended beneficial effects (Weese and Arroyo, 2003). In this regard, additional probiotic supplements are required to maintain and improve canine health.

Kefir is a traditional dairy food product produced easily via natural fermentation of milk with kefir grain at room temperature, requiring no special instrument to acquire the final product (Guzel-Seydim et al., 2011). Hence, kefir is widely produced and consumed at home (Guzel-Seydim et al., 2011). This study indicated that all dogs consumed kefir spontaneously and that they preferred consuming kefir even in the mid and late experimental periods, which is considered a desirable feature for an optimal pet treat (Weese and Arroyo, 2003). Some studies report that probiotic microorganisms should naturally originate from the target host species; however, the present study shows that probiotics of another origin can be successfully applied to improve the gut microbiota of healthy dogs as well (Kailasapathy and Chin, 2000).

We previously reported that kefir and its associated microorganisms exerted broad-spectrum and significant modulatory effects on gut microbiota in healthy mice and in mouse models of disease (Kim et al. 2015b, 2017a,b; Jeong et al., 2017b). Concurrently, 2 wk of kefir consumption clearly altered gut microbiota of dogs at the phylum, family, and species levels. Gut microbiota compositions are unique and stable in each individual, especially in adults (Tannock, 2005). It is remarkable that although all dogs included herein were adults and varied in age and sex, kefir significantly modulated the composition of their gut microbiota. The NGS-based analysis revealed that kefir consumption significantly decreased the relative abundance of the phylum Fusobacteria and the families Clostridiaceae, Fusobacteriaceae, and Ruminococcaceae and significantly increased the relative abundance of the families Prevotellacea, Selenomonadaceae, and Sutterellaceae. At the species level, the relative abundance of Bc. uniformis, Fb. perfoetens, Fc. prausnitzii, Fb. mortiferum, Ps. capillosus, and Fs. saccharivorans decreased, whereas that of $C$. mitsuokai increased. Moreover, qPCR analysis revealed that kefir consumption significantly increased the relative abundance of lactic acid bacteria, decreased the Firmicutes:Bacteroidetes ratio, and increased the lactic acid bacteria:Enterobacteriaceae ratio, which was consistent with the results obtained by NGS analysis.
A rather remarkable finding is the reduction in the relative abundance of the phylum Fusobacteria, revealed via NGS-based analysis. Fusobacteria comprises gramnegative, nonsporulating, obligate anaerobic bacilli. A recent study reported in vitro antimicrobial activity of kefir against Fusobacterium nucleatum, a well-known member of phylum Fusobacteria (Guzel-Seydim et al., 2016). In parallel, we reported a negative interaction between kefir and intestinal phylum Fusobacteria and family Fusobacteriaceae in vivo, more specifically with $F b$. perfoetens and Fb. mortiferum. The phylum Fusobacteria appears to be a major member of commensal gut microbiota in dogs, and a recent study suggested that this microbial group was not associated with inflammatory bowel diseases in dogs, which is opposite in humans (Vázquez-Baeza et al., 2016). As the physiological roles of this bacterium in canine gut health are not yet entirely understood, future studies should find whether the decrease of Fusobacteria by probiotic consumption in healthy dogs is beneficial or harmful.

Another interesting finding is an increase in the lactic acid bacteria:Enterobacteriaceae ratio revealed via $\mathrm{qPCR}$ assay. Lactic acid bacteria acidify the host gut by producing various organic acids, creating an unfavorable environment for acid-sensitive members of family Enterobacteriaceae (Edelson-Mammel et al., 2006; Dharmasena et al., 2016). In addition, lactic acid bacteria associated with kefir compete with members of the family Enterobacteriaceae in the host for nutrition and adhesion sites and produce a bacteriocin or antimicrobial exopolysaccharides to counter them (Powell et al., 2007; Miao et al., 2014; Jeong et al., 2017a). However, the family Enterobacteriaceae was not significantly reduced after kefir consumption with both NGS analysis and qPCR assay in the present study. The increase of the lactic acid bacteria:Enterobacteriaceae ratio could be solely attributed to the increase of lactic acid bacteria population by the inflow of kefir-originated lactic acid bacteria. Given that the population of Enterobacteriaceae has a significant correlation with inflammatory bowel diseases in dogs (Vázquez-Baeza et al., 2016), further studies are warranted to control the Enterobacteriaceae population by kefir consumption with some modifications (i.e., increased amount or frequency of kefir consumption, use of kefir from other origins, and use of its sole microorganism).

A final aspect regarding the modulatory effects of kefir on canine gut microbiota is the reduction in the Firmicutes:Bacteroidetes ratio and the relative abundance of $F c$. prausnitzii. Both intestinal microbial biomarkers are associated with metabolic diseases such as obesity and hepatic steatosis because these microorganisms are related to the host energy harvest from the 
intestinal contents (Balamurugan et al., 2010; Kim et al., 2018). The exact roles and contribution of these microbial members in relation to host health and diseases remain a matter of debate; for example, the Firmicutes: Bacteroidetes ratio was first believed to be positively correlated with obesity, whereas some current studies have failed to demonstrate the correlation between Firmicutes:Bacteroidetes ratio and weight gain and body mass index in human (Koliada et al., 2017). The clinical significance of this microbial marker is also unclear in dogs. There are only a few studies on the association of this marker with canine health, and one study revealed that a high-protein and low-carbohydrate diet increased the Firmicutes:Bacteroidetes ratio in dogs ( $\mathrm{Li}$ et al., 2017). For Fc. prausnitzii, it was reported that obese children harbored a higher number of this species compared with nonobese participants (Balamurugan et al., 2010), and this bacterium played a role in alleviating low-grade inflammation in metabolic diseases (Furet et al., 2010). In dogs, the reduction of Fc. prausnitzii was observed in dogs with nonhemorrhagic diarrhea, acute hemorrhagic diarrhea, and active inflammatory bowel diseases (Suchodolski et al., 2012). In contrast, in a fiber-deprived environment, this species is able to digest the mucin layer, which provides a more favorable environment for opportunistic pathogens to penetrate the host gut barrier (Velasquez-Manoff, 2015). Given the multifaceted roles of these gut microbial members in various situations, the results obtained in the present study need to be interpreted with caution. In addition, the effect of kefir consumption on microbiota and weight gain needs to be studied in overweight or obese dogs in the future.

Furthermore, significant changes reported in other intestinal microbial taxa including Ruminococcaceae, Selenomonadaceae, and Sutterellaceae and their clinical and physiological significance have rarely been studied in both human and dogs; hence, further studies are warranted to understand the significance of changes in these taxa. The relative abundance of gut yeast remained unchanged upon qPCR analysis, although kefir contains a large population of probiotic yeast (Kim et al., 2015a). Despite the total number of yeast remaining unchanged, yeast composition at lower phylogenetic levels may have been altered, which should be addressed in future studies.

We successfully demonstrated that kefir modulates the gut microbiota of healthy adult dogs within $2 \mathrm{wk}$ of consumption without any harmful effects on analysis of BW, BCS, fecal occult blood, hematological parameters, and dietary habits. The 16S rDNA ampliconbased community analysis and qPCR assay of fecal samples revealed that kefir consumption clearly altered the composition of gut microbiota at different levels of taxa. Therefore, kefir may be applied as a novel functional pet food supplement to improve the quality of life of dogs, much to their owners' satisfaction.

\section{ACKNOWLEDGMENTS}

This work was supported by the Strategic Initiative for Microbiomes in Agriculture and Food, Ministry of Agriculture, Food and Rural Affairs, Republic of Korea (as part of the multiministerial Genome Technology to Business Translation Program; grant number 9180152 ). This research was also supported by Basic Science Research Program through the National Research Foundation of Korea (NRF) funded by the Ministry of Education (NRF-2018R1D1A1B07049710). In addition, this work was supported by the KU Research Professor Program of Konkuk University.

\section{REFERENCES}

Aktas, M. S., M. K. Borku, and Y. Ozkanlar. 2007. Efficacy of Saccharomyces boulardii as a probiotic in dogs. Bull. Vet. Inst. Pulawy 51:365-369.

Asanuma, N., M. Kawato, and T. Hino. 2001. Presence of Butyrivibrio fibrisolvens in the digestive tract of dogs and cats, and its contribution to butyrate production. J. Gen. Appl. Microbiol. 47:313-319.

Balamurugan, R., G. George, J. Kabeerdoss, J. Hepsiba, A. M. Chandragunasekaran, and B. S. Ramakrishna. 2010. Quantitative differences in intestinal Faecalibacterium prausnitzii in obese Indian children. Br. J. Nutr. 103:335-338.

Beasley, S. S., T. J. K. Manninen, and P. R. J. Saris. 2006. Lactic acid bacteria isolated from canine faeces. J. Appl. Microbiol. 101:131-138.

Bourrie, B. C. T., B. P. Willing, and P. D. Cotter. 2016. The microbiota and health promoting characteristics of the fermented beverage kefir. Front. Microbiol. 7:647. https://doi.org/10.3389/fmicb 2016.00647.

Caporaso, J. G., J. Kuczynski, J. Stombaugh, K. Bittinger, F. D. Bushman, E. K. Costello, N. Fierer, A. G. Pena, J. K. Goodrich, J. I. Gordon, and G. A. Huttley. 2010. QIIME allows analysis of highthroughput community sequencing data. Nat. Methods 7:335-336.

Codex Alimentarius. 2003. Codex-Stan CXS 243-2003; Codex Standard for Fermented Milks. Accessed October 10, 2018. http://www .fao.org/fao-who-codexalimentarius/en/.

Dharmasena, M. N., C. M. Feuille, C. E. C. Starke, A. A. Bhagwat, S. Stibitz, and D. J. Kopecko. 2016. Development of an acid-resistant Salmonella typhi Ty21a attenuated vector for improved oral vaccine delivery. PLoS One 11:e0163511.

Edelson-Mammel, S., M. K. Porteous, and R. L. Buchanan. 2006. Acid resistance of twelve strains of Enterobacter sakazakii, and the impact of habituating the cells to an acidic environment. J. Food Sci. 71:M201-M207.

Furet, J. P., L. C. Kong, J. Tap, C. Poitou, A. Basdevant, J. L. Bouillot, D. Marial, G. Corthier, J. Dore, C. Henegar, S. Rizkalla, and K. Clement. 2010. Differential adaptation of human gut microbiota to bariatric surgery-induced weight loss: Links with metabolic and low-grade inflammation markers. Diabetes 59:3049-3057.

Guzel-Seydim, Z. B., M. Dibekci, E. Cagdas, and A. C. Seydim. 2016. Effect of kefir on Fusobacterium nucleatum in potentially preventing intestinal cancer. Funct. Food Health Dis. 6:469-477.

Guzel-Seydim, Z. B., T. Kok-Tas, A. K. Greene, and A. C. Seydim. 2011. Review: Functional properties of kefir. Crit. Rev. Food Sci. Nutr. 51:261-268. 
Hooda, S., Y. Minamoto, J. S. Suchodolski, and K. S. Swanson. 2012. Current state of knowledge: The canine gastrointestinal microbiome. Anim. Health Res. Rev. 13:78-88.

Jeong, D., D. H. Kim, I. B. Kang, H. Kim, K. Y. Song, H. S. Kim, and K. H. Seo. 2017a. Characterization and antibacterial activity of a novel exopolysaccharide produced by Lactobacillus kefiranofaciens DN1 isolated from kefir. Food Control 78:436-442.

Jeong, D., D. H. Kim, I. B. Kang, H. Kim, K. Y. Song, H. S. Kim, and K. H. Seo. 2017b. Modulation of gut microbiota and increase in fecal water content in mice induced by administration of Lactobacillus kefiranofaciens DN1. Food Funct. 8:680-686.

Kailasapathy, K., and J. Chin. 2000. Survival and therapeutic potential of probiotic organisms with reference to Lactobacillus acidophilus and Bifidobacterium spp. Immunol. Cell Biol. 78:80-88.

Kim, D. H., J. W. Chon, H. Kim, H. S. Kim, D. Choi, D. G. Hwang, and K. H. Seo. 2015a. Detection and enumeration of lactic acid bacteria, acetic acid bacteria and yeast in kefir grain and milk using quantitative real-time PCR. J. Food Saf. 35:102-107.

Kim, D. H., J. W. Chon, H. Kim, and K. H. Seo. 2015b. Modulation of intestinal microbiota in mice by kefir administration. Food Sci. Biotechnol. 24:1397-1403.

Kim, D. H., D. Jeong, I. B. Kang, H. Kim, K. Y. Song, and K. H. Seo. 2017a. Dual function of Lactobacillus kefiri DH5 in preventing high-fat-diet-induced obesity: Direct reduction of cholesterol and upregulation of PPAR-a in adipose tissue. Mol. Nutr. Food Res. 61:1700252.

Kim, D. H., D. Jeong, H. Kim, and K. H. Seo. 2018. Modern perspectives on the health benefits of kefir in next generation sequencing era: Improvement of the host gut microbiota. Crit. Rev. Food Sci. Nutr. 16:1-12.

Kim, D. H., H. Kim, D. Jeong, I. B. Kang, J. W. Chon, H. S. Kim, K. Y. Song, and K. H. Seo. 2017b. Kefir alleviates obesity and hepatic steatosis in high-fat diet-fed mice by modulation of gut microbiota and mycobiota: Targeted and untargeted community analysis with correlation of biomarkers. J. Nutr. Biochem. 44:35-43.

Koliada, A., G. Syzenko, V. Moseiko, L. Budovska, K. Puchkov, V. Perederiy, Y. Gavalko, A. Dorofeyev, M. Romanenko, S. Tkach, L. Sineok, O. Lushchak, and A. Vaiserman. 2017. Association between body mass index and Firmicutes/Bacteroidetes ratio in an adult Ukrainian population. BMC Microbiol. 17:120.

Lewis, S. J., and K. W. Heaton. 1997. Stool form as a useful guide to intestinal transit time. Scand. J. Gastroenterol. 32:920-924

Li, Q., C. L. Lauber, G. Czarnecki-Maulden, Y. Pan, and S. S. Hannah. 2017. Effects of the dietary protein and carbohydrate ratio on gut microbiomes in dogs of different body conditions. MBio 8:e01703-e01716.

Li, W., L. Fu, B. Niu, S. Wu, and J. Wooley. 2012. Ultrafast clustering algorithms for metagenomic sequence analysis. Brief. Bioinform. 13:656-668.

Magoč, T., and S. L. Salzberg. 2011. FLASH: Fast length adjustment of short reads to improve genome assemblies. Bioinformatics 27:2957-2963.

Manninen, T. J., M. L. Rinkinen, S. S. Beasley, and P. E. Saris. 2006. Alteration of the canine small-intestinal lactic acid bacterium microbiota by feeding of potential probiotics. Appl. Environ. Microbiol. 72:6539-6543.

Miao, J. Y., H. X. Guo, Y. W. Ou, G. Liu, X. Fang, Z. L. Liao, C. Ke, Y. J. Chen, L. C. Zhao, and Y. Cao. 2014. Purification and characterization of bacteriocin $\mathrm{F} 1$, a novel bacteriocin produced by Lactobacillus paracasei subsp. tolerans FX-6 from Tibetan kefir, a traditional fermented milk from Tibet, China. Food Control $42: 48-53$

Powell, J. E., R. C. Witthuhn, S. D. Todorov, and L. M. T. Dicks, 2007. Characterization of bacteriocin ST8KF produced by a kefir isolate Lactobacillus plantarum ST8KF. Int. Dairy J. 17:190-198.
Rinkinen, M., K. Jalava, E. Westermarck, S. Salminen, and A. C. Ouwehand. 2003. Interaction between probiotic lactic acid bacteria and canine enteric pathogens: A risk factor for intestinal Enterococcus faecium colonization? Vet. Microbiol. 92:111-119.

Rossi, G., G. Pengo, M. Caldin, A. P. Piccionello, J. M. Steiner, N. D. Cohen, A. E. Jergens, and J. S. Suchodolski. 2014. Comparison of microbiological, histological, and immunomodulatory parameters in response to treatment with either combination therapy with prednisone and metronidazole or probiotic VSL\#3 strains in dogs with idiopathic inflammatory bowel disease. PLoS One 9. https:// doi.org/10.1371/journal.pone.0094699.

Schmitz, S., and J. Suchodolski. 2016. Understanding the canine intestinal microbiota and its modification by pro-, pre- and synbiotics - What is the evidence? Vet. Med. Sci. 2:71-94.

Silva, B. C., L. R. C. Jung, S. H. C. Sandes, L. B. Alvim, M. R. Q. Bomfim, J. R. Nicoli, E. Neumann, and A. C. Nunes. 2013. In vitro assessment of functional properties of lactic acid bacteria isolated from faecal microbiota of healthy dogs for potential use as probiotics. Benef. Microbes 4:267-275.

Strompfová, V., A. Lauková, and A. C. Ouwehand. 2004. Lactobacilli and enterococci-Potential probiotics for dogs. Folia Microbiol. (Praha) 49:203-207.

Strompfová, V., M. Marciňáková, M. Simonová, B. Bogovič-Matijašić, and A. Lauková. 2006. Application of potential probiotic Lactobacillus fermentum AD1 strain in healthy dogs. Anaerobe 12:75-79.

Strompfová, V., M. P. Simonová, S. Gancarčíková, D. Mudroňová, J. Farbáková, A. Mad'ari, and A. Lauková. 2014. Effect of Bifidobacterium animalis $\mathrm{B} / 12$ administration in healthy dogs. Anaerobe 28:37-43

Suchodolski, J. S. 2011. Intestinal microbiota of dogs and cats: A bigger world than we thought. Vet. Clin. North Am. Small Anim. Pract. 41:261-272.

Suchodolski, J. S., M. E. Markel, J. F. Garcia-Mazcorro, S. Unterer, R. M. Heilmann, S. E. Dowd, P. Kachroo, I. Ivanov, Y. Minamoto, E. M. Dillman, J. M. Steiner, A. K. Cook, and L. Toresson. 2012. The fecal microbiome in dogs with acute diarrhea and idiopathic inflammatory bowel disease. PLoS One 7:e51907. https://doi.org/ 10.1371/journal.pone.0051907.

Suchodolski, J. S., and K. Simpson. 2013. Canine gastrointestinal microbiome in health and disease. Vet. Focus 23:22-28.

Tang, Y., and P. E. J. Saris. 2013. Strain-specific detection of orally administered canine jejunum-dominated Lactobacillus acidophilus LAB20 in dog faeces by real-time PCR targeted to the novel surface layer protein. Lett. Appl. Microbiol. 57:330-335.

Tannock, G. W. 2005. New perceptions of the gut microbiota: Implications for future research. Gastroenterol. Clin. North Am. 34:361-382.

Vázquez-Baeza, Y., E. R. Hyde, J. S. Suchodolski, and R. Knight. 2016. Dog and human inflammatory bowel disease rely on overlapping yet distinct dysbiosis networks. Nat. Microbiol. 1:16177.

Velasquez-Manoff, M. 2015. Gut microbiome: The peacekeepers. Nature 518(7540):S3-S11. https://doi.org/10.1038/518S3a.

Weese, J. S., and M. E. Anderson. 2002. Preliminary evaluation of Lactobacillus rhamnosus strain GG, a potential probiotic in dogs. Can. Vet. J. 43:771-774.

Weese, J. S., and L. Arroyo. 2003. Bacteriological evaluation of dog and cat diets that claim to contain probiotics. Can. Vet. J. 44:212216

Wynn, S. G. 2009. Probiotics in veterinary practice. J. Am. Vet. Med. Assoc. 234:606-613.

Zhang, Z., S. Schwartz, L. Wagner, and W. Miller. 2000. A greedy algorithm for aligning DNA sequences. J. Comput. Biol. 7:203-214. 